\title{
NEUROLOGÍA: LA CURA DEL ALZHEIMER. DE LA FICCIÓN A LA REALIDAD. EL ORIGEN DEL PLANETA DE LOS SIMIOS (2011)
}

\section{Neurology: Alzheimer cure: From fiction to reality. Rise of the planet of apes (2011)}

Natalin MORENO-CHINCHAY; Josue RAMIREZ-ORELLANA

Facultad de Medicina Humana. Universidad Norbert Wiener (Perú).

Correo electrónico: natiliz27@hotmail.com

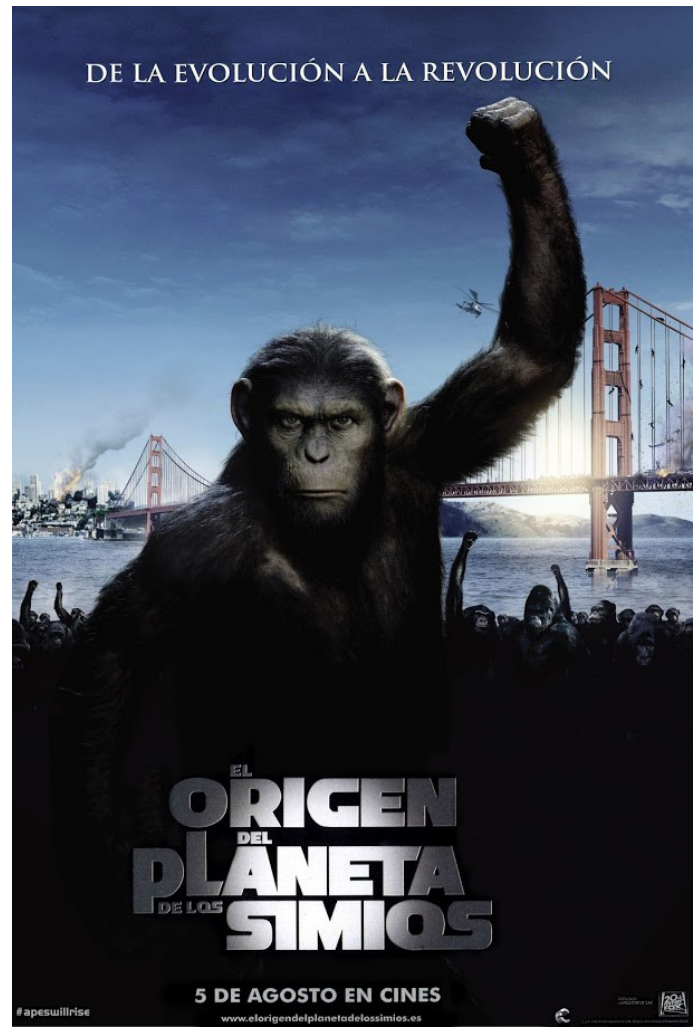

Acción: San Francisco (EEUU). Época actual imaginaria. Cartel español.
Ficha técnica

Título original: Rise of the planet of apes.

País: EEUU.

Año: 2011.

Director: Rupert Wyatt.

Música: Patrick Doyle.

Fotografía: Andrew Lesnie.

Montaje: Conrad Buff IV, Mark Goldblatt.

Guión: Rick Jaffa y Amanda Silver. Basada en la novela de Piere Boulle, La Planète des Singes (1963).

Intérpretes: Andy Derkis, Karin Konoval, Terry Notary, Richard Ridings, Christopher Gordon, Devyn Dalton, Jay Caputo, James Franco, Freida Pinto, John Lithgow, Brian Cox, Tom Felton,...

Color: color.

Duración: 110 minutos.

Género: ciencia ficción, aventura, acción.

Idioma original: inglés.

Productoras: 20th Century Fox, Dune Entertainment, Chernin Entertainment, Ingenious Media, Big Screen Productions, Ingenious Film Partners, Dune Entertainment III.

Sinopsis: "Precuela del ya mítico largometraje "El planeta de los simios". Will Rodman (James Franco) es un joven científico que está investigando con monos para obtener un tratamiento

Rev. Med. Cine. 2020; 16(2), 135-141 Ediciones Universidad de Salamanca / @®@ J. Med. Mov., 2020; 16 (2), $135-141$ 


\section{NEUROLOGÍA: LA CURA DEL ALZHEIMER. DE LA FICCIÓN A LA REALIDAD. \\ EL ORIGEN DEL PLANETA DE LOS SIMIOS (2011) \\ NATALIN MORENO-CHINCHAY; JOSUE RAMIREZ-ORELLANA}

contra el Alzheimer, una enfermedad que afecta a su padre (John Lithgow). Uno de esos primates, César, un chimpancé recién nacido al que Will se llevó a casa para protegerlo, experimenta una evolución en su inteligencia verdaderamente sorprendente. En el estudio del simio le ayudará una bella primatóloga Ilamada Caroline (Freida Pinto)» FilmAffinity.
Comentario: Séptima película de la saga.

Disponibilidad: El origen del planeta de los simios (DVD. Blu-Ray). Madrid: 20th Century Fox; 2011.

\section{Enlaces:}

$\mathrm{https} / / / w w w . i m d b . c o m / t i t l e / t t 1318514 /$ ?ref_=ttfc_ fc_tt

https://www.filmaffinity.com/pe/film793766. html

\section{Trailer}

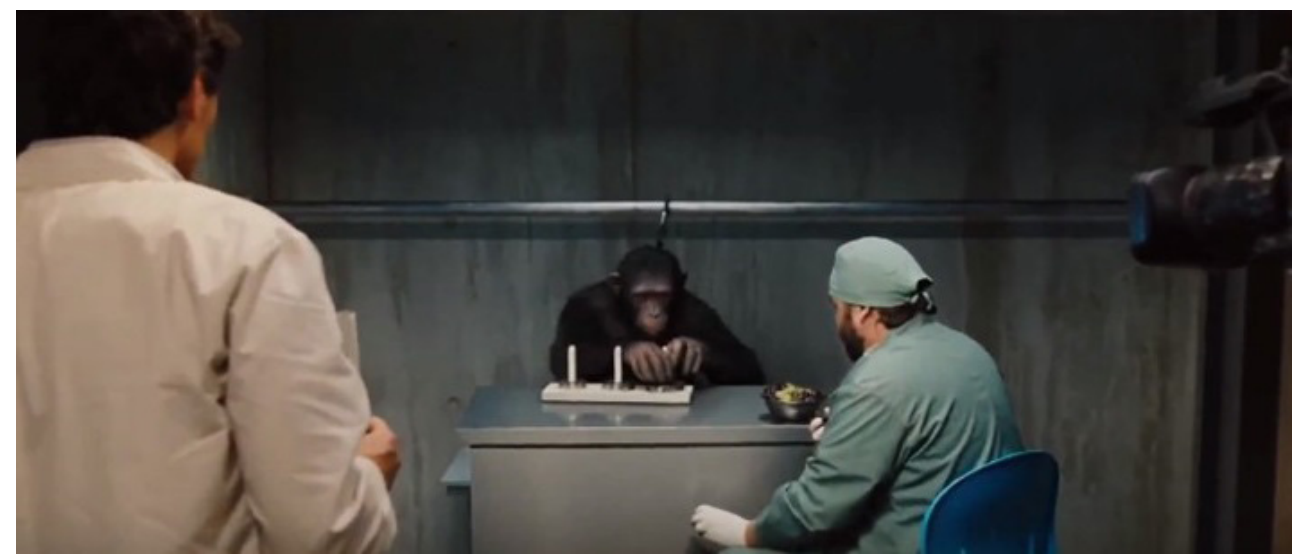

Simio es evaluado mediante la torre de Lucas.

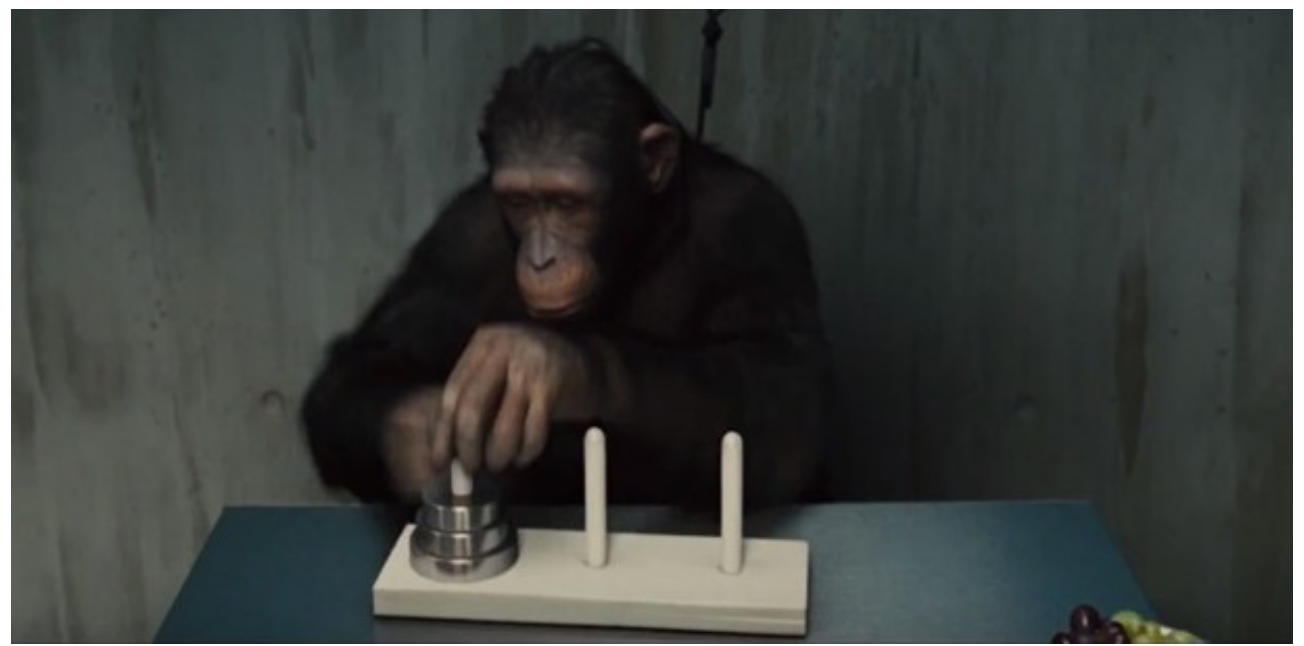

El simio logra realizar la torre de Lucas en 20 movimientos, siendo el récord de 15 movimientos.

Rev. Med. Cine. 2020; 16(2), 135-141 Ediciones Universidad de Salamanca /

J. Med. Mov., 2020; 16 (2), 135-141 
NEUROLOGÍA: LA CURA DEL ALZHEIMER. DE LA FICCIÓN A LA REALIDAD.

EL ORIGEN DEL PLANETA DE LOS SIMIOS (2011)

NATALIN MORENO-CHINCHAY; JOSUE RAMIREZ-ORELLANA

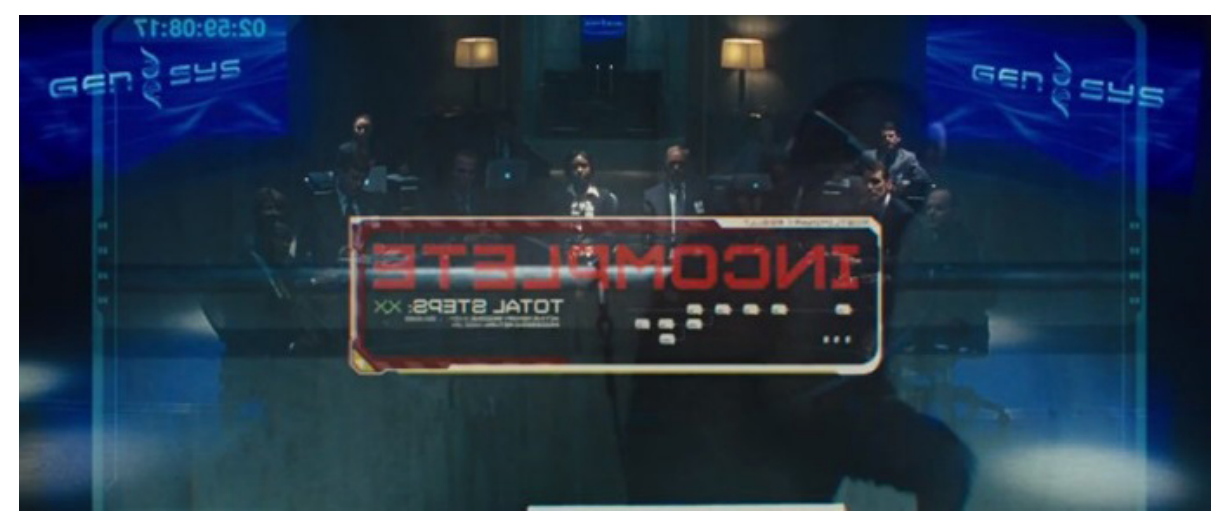

Antes de administrarle la terapia genética llamada ALZ 112, el simio no podía realizar la prueba de la torre de Lucas exitosamente.

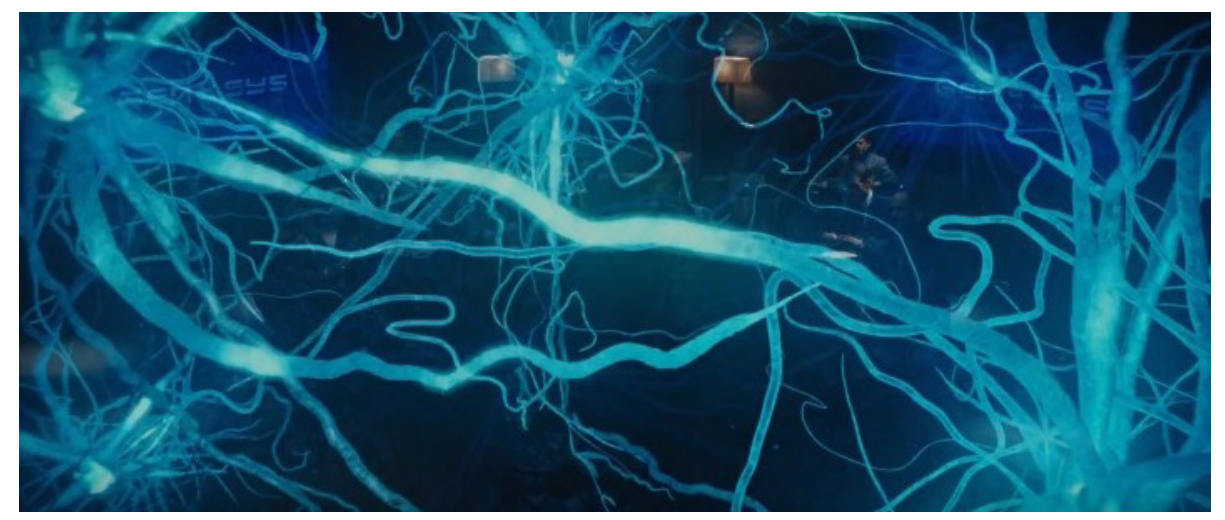

Se explica la terapia. Dr Will: «Suministramos algo llamado ALZ-112, una terapia genética que le permite al cerebro crear sus propias células para regenerarse, en términos biológicos se le llama neurogénesis» (minuto 5.02).

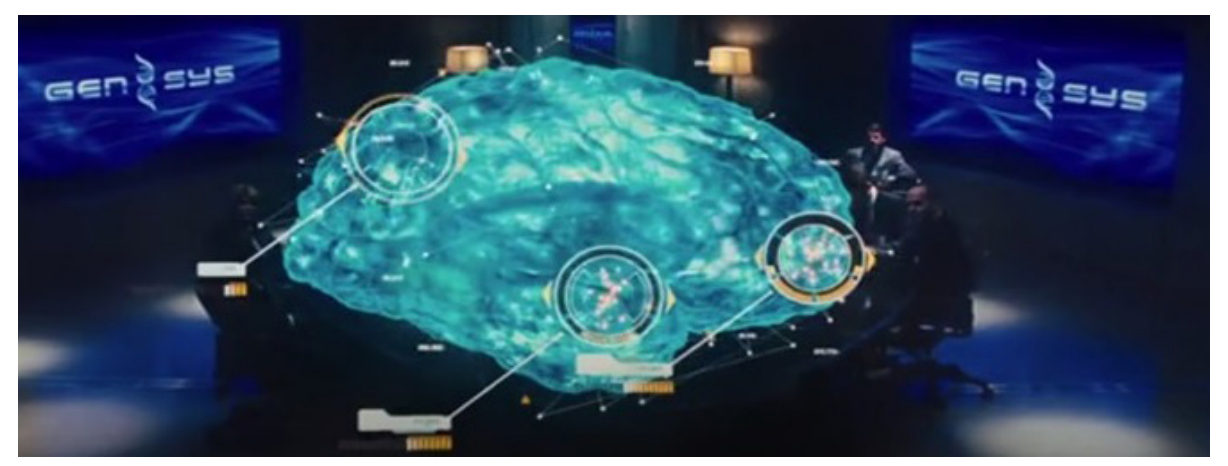

Se muestran las regiones cerebrales en donde la terapia estimularía la neurogénesis.

Rev. Med. Cine. 2020; 16(2), 135-141 Ediciones Universidad de Salamanca / @®@ J. Med. Mov., 2020; 16 (2), $135-141$ [ 137 ] 
NEUROLOGÍA: LA CURA DEL ALZHEIMER. DE LA FICCIÓN A LA REALIDAD.

EL ORIGEN DEL PLANETA DE LOS SIMIOS (2011)

NATALIN MORENO-CHINCHAY; JOSUE RAMIREZ-ORELLANA

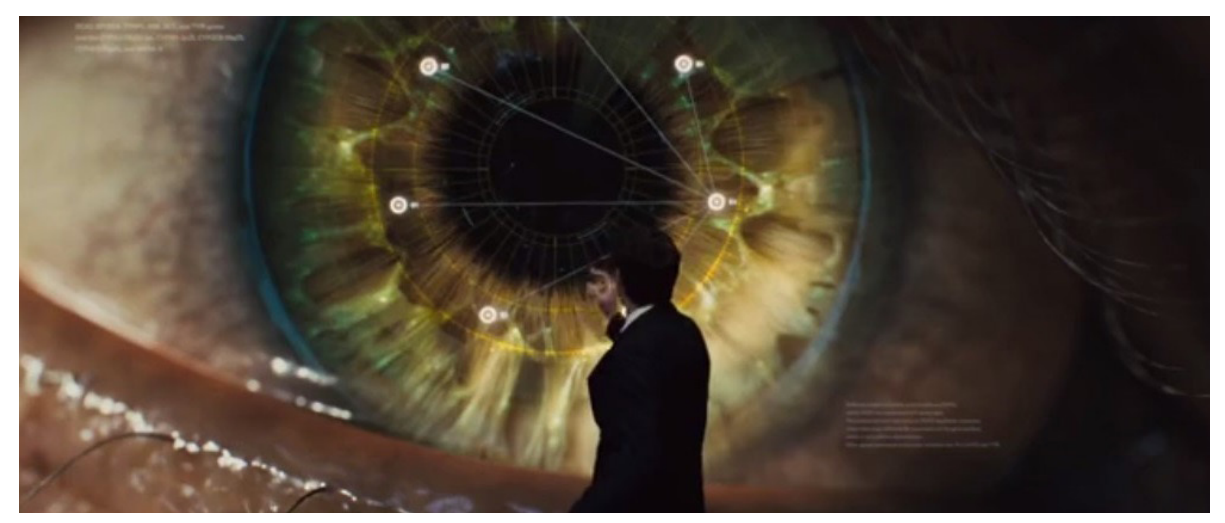

Presencia de destellos verdes en el iris, presentándose como único efecto adverso de la terapia.

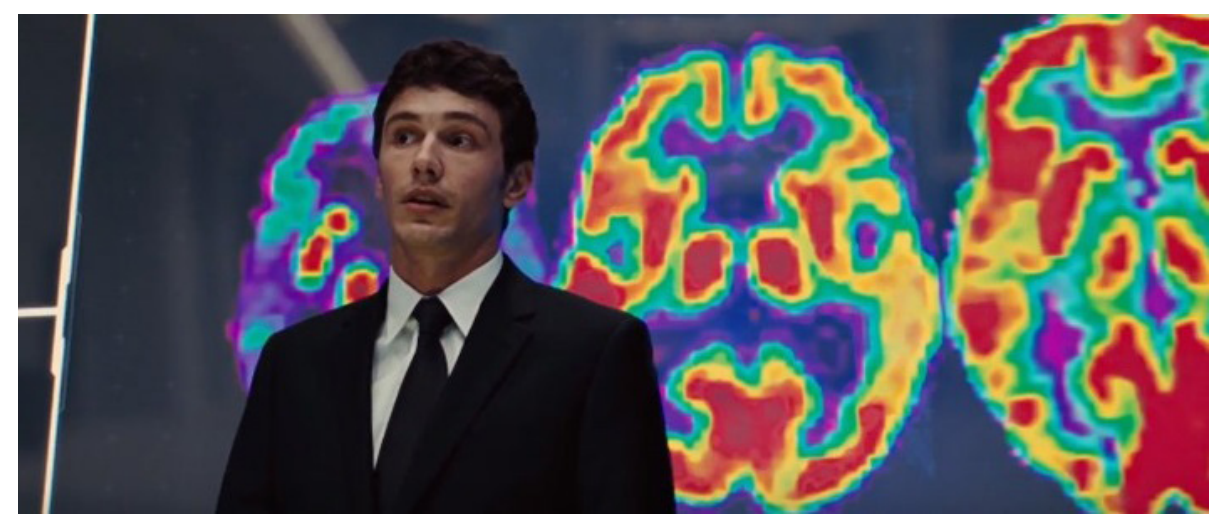

Se concluye con el propósito final de la terapia con el ALZ-112. Dr. Will: "Aquí en GENSYS se le llama, la cura del Alzheimer» (minuto 5.18).

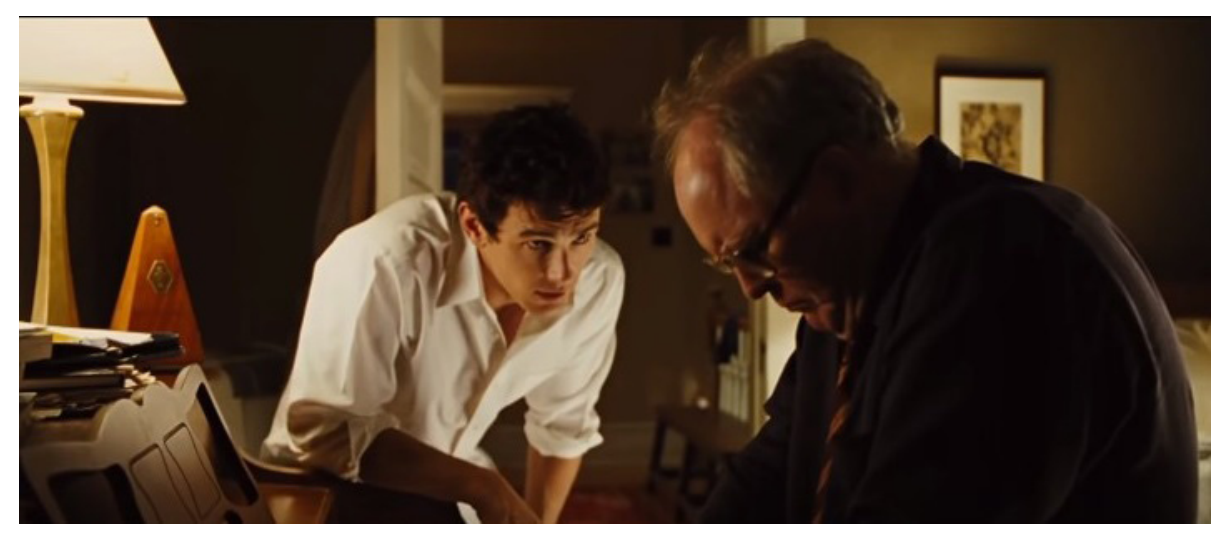

El padre del Dr. Will padece de la enfermedad de Alzheimer (EA).

Rev. Med. Cine. 2020; 16(2), 135-141 Ediciones Universidad de Salamanca / @@@

J. Med. Mov., 2020; 16 (2), 135-141 
NEUROLOGÍA: LA CURA DEL ALZHEIMER. DE LA FICCIÓN A LA REALIDAD.

EL ORIGEN DEL PLANETA DE LOS SIMIOS (2011)

NATALIN MORENO-CHINCHAY; JOSUE RAMIREZ-ORELLANA

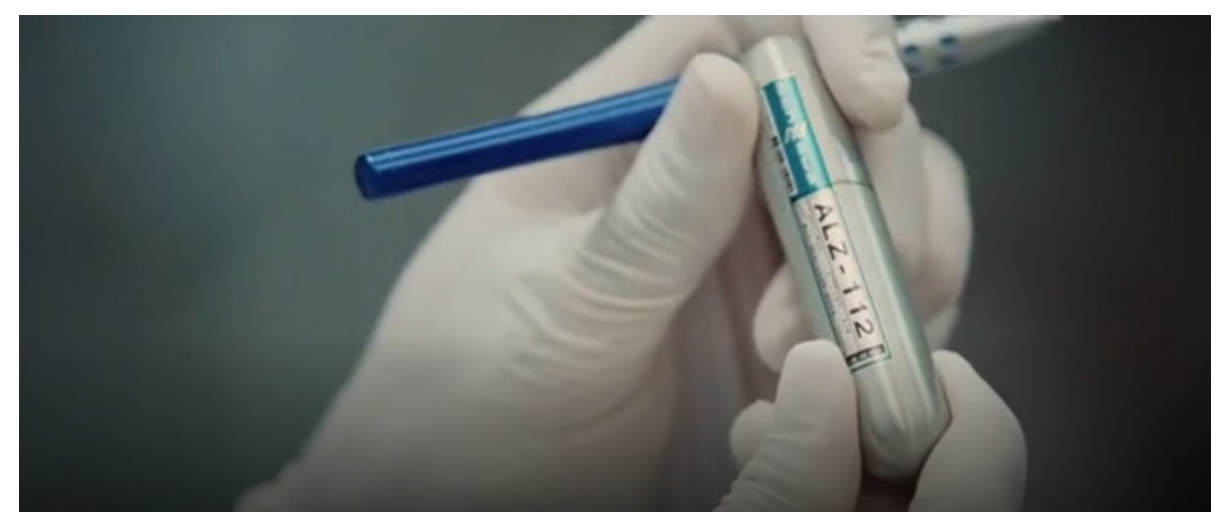

El Dr. Will roba el ALZ 112 para probarlo en su padre.

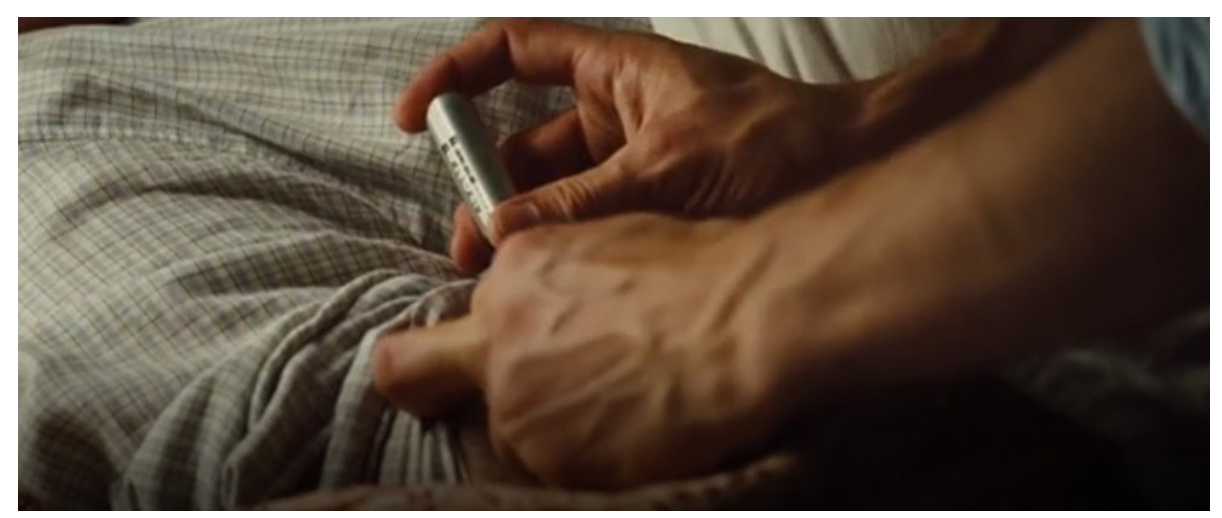

Aplicación del medicamento vía parenteral.

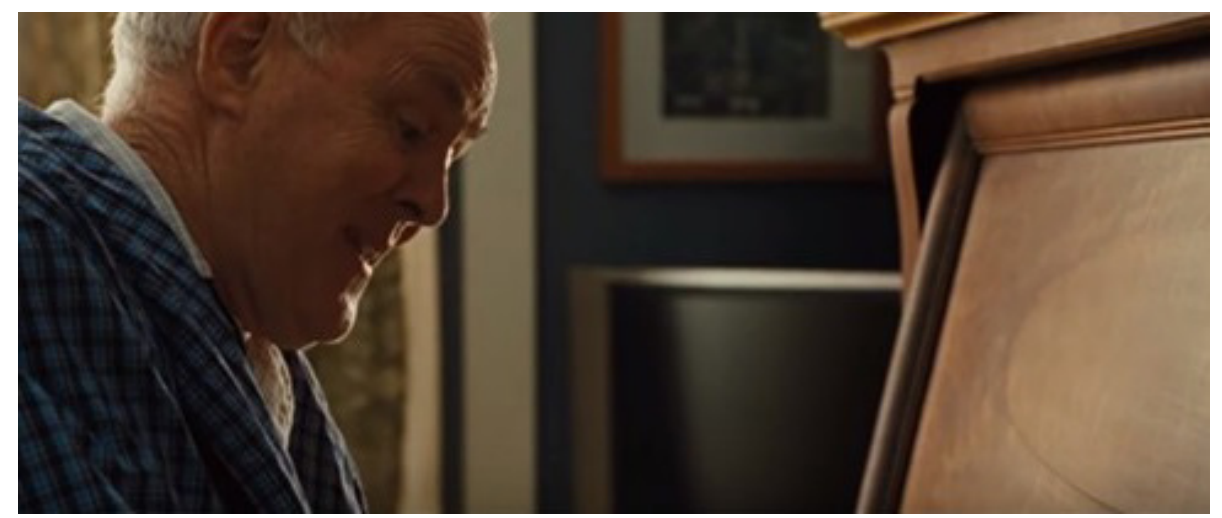

Después de la administración del ALZ 112, el padre del Dr. Will muestra señales de mejoría en los signos de la EA.

Rev. Med. Cine. 2020; 16(2), 135-141 Ediciones Universidad de Salamanca / @®@ J. Med. Mov., 2020; 16 (2), $135-141$ [139] 


\section{NEUROLOGÍA: LA CURA DEL ALZHEIMER. DE LA FICCIÓN A LA REALIDAD. EL ORIGEN DEL PLANETA DE LOS SIMIOS (2011) NATALIN MORENO-CHINCHAY; JOSUE RAMIREZ-ORELLANA}

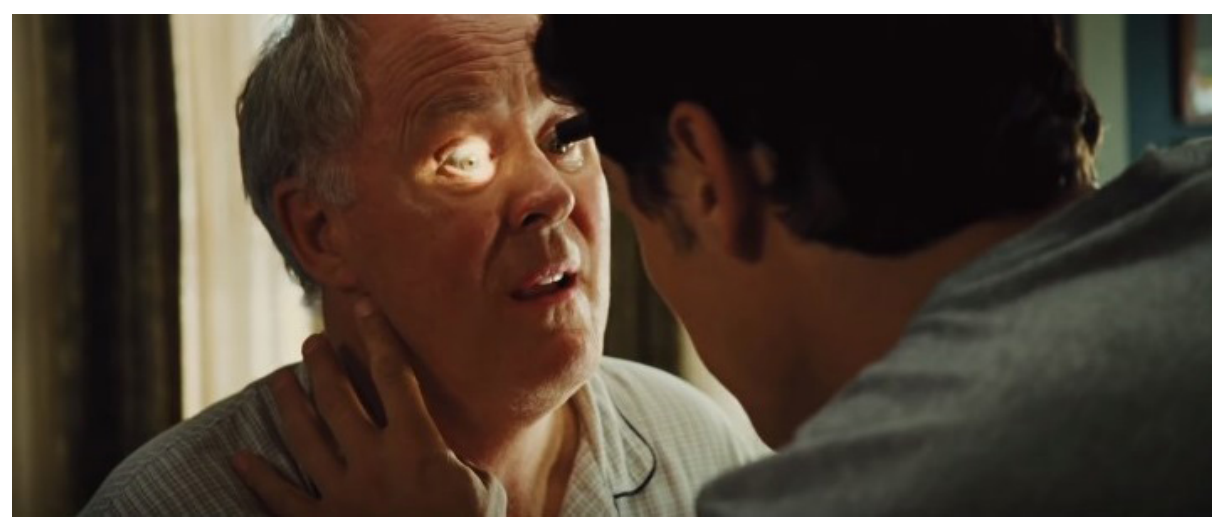

Evaluación de cambios en la coloración en el iris, demostrando la acción del ALZ -112, tal como se mostraba en el simio.

El término Neurogénesis mencionada en la película, posee una estrecha relación con la realidad actual a cerca de los avances en el tratamiento de la enfermedad de Alzheimer (EA), tal como lo demuestra Mu y Gage (2011): «We have presented evidence that multiple molecular players known to contribute to $A D$ pathogenesis, mainly ApoE, PS1, APP and its metabolites, can modulate adult hippocampal neurogenesis» ${ }^{1}$. Según otras investigaciones realizadas por Fuentes y Mena (2009): «El factor de crecimiento neuronal y el factor neurotrófico derivado del cerebro (NGF y BDNF, por sus siglas en inglés, respectivamente) promueven la sobrevida de grupos neuronales importantes en la $E A$, tales como los de algunas regiones del cerebro anterior, el hipocampo y la neocorteza» ${ }^{2}$, siendo justamente dichas áreas mencionadas en la escena donde el Dr Will expone sobre la terapia, llevándonos a pensar que la realidad fue plasmada en la película de ciencia ficción.

En la actualidad, un tratamiento eficaz para la cura de la EA no es una idea tan lejana, pues un estudio de Plosker y Gauthier (2009) mostraron que: «Cerebrolysin mejoró la viabilidad de neuronas cultivadas in vitro y rescató neuronas colinérgicas mediales septales posterior a la administración intraperitoneal en ratas al realizar cortes en la fimbria-fórnix en el cerebro» ${ }^{3}$, esto se puede llegar a complementar junto a un artículo en donde Fuentes y Mena indican que mediante el uso de cerebrolisina: "Se ha encontrado que hay una disminución del proceso de apoptosis y de actividad de la enzima caspasa-3 en las células neuronales progenitoras ${ }^{2}$, la cual es una enzima que induce a la apoptosis neuronal debido a una posible activación excesiva.

Además de existir una serie de medicamentos que podrían ayudar en la neurogénesis, existen también otras maneras de producirla, se habla mucho de qué factores medioambientales tendrían un gran impacto frente a este fenómeno. Es así como lo muestra Fernández-Verdecia, et al. (2009): «Un factor medioambiental con posibles propiedades neuroprotectoras y neurogénicas para la EA son los cambios en el estilo de vida a través de la dieta [...] La curcumina la cual induce neurogénesis en el hipocampo del cerebro adulto, efecto que se asocia a la activación del ERK y la cinasa p38, que son vías de transducción de la señalización intracelular, las cuales están implicadas en la regulación de la plasticidad neuronal y la respuesta al estrés. Los inhibidores del ERK y la cinasa p38 bloquean el efecto mitogénico de la curcumina en las células progenitoras neurales; así, el suministro de curcumina amplía la neurogénesis hipocampal adulta e impide la muerte de

Rev. Med. Cine. 2020; 16(2), 135-141 Ediciones Universidad de Salamanca / @@ J. Med. Mov., 2020; 16 (2), $135-141$ 


\section{NEUROLOGÍA: LA CURA DEL ALZHEIMER. DE LA FICCIÓN A LA REALIDAD. EL ORIGEN DEL PLANETA DE LOS SIMIOS (2011) \\ NATALIN MORENO-CHINCHAY; JOSUE RAMIREZ-ORELLANA}

neuronas en modelos animales de enfermedad neurodegenerativa» ${ }^{4}$.

En el último año se han realizado avances importantes en el desarrollo de la neurogénesis, Kempermann y Boldrini citado por Steiner, et al. (2019): "Several studies have demonstrated the presence of immature neurons throughout life in the human hippocampus, although the quantitative estimates have slightly varied $»^{5}$. Esto nos podría dar indicios de la existencia de regiones de reserva neuronal para un futuro requerimiento de células nuevas.

En conclusión, la película muestra un conocimiento acerca de la terapéutica actual de la $\mathrm{EA}$, demostrando que posee una gran base científica al reflejar una posible cura para la misma. Además, se refleja una relación entre la ficción y realidad refiriéndose al intento y constancia en la búsqueda de una terapia para las enfermedades neurodegenerativas como lo es la EA. Las diversas investigaciones que existen acerca de posibles curas, nos dan esperanza que en un futuro no muy lejano se pueda remediar los problemas neurodegenerativos.

\section{Referencias}

1. Mu Y, Gage F. Adult hippocampal neurogenesis and its role in Alzheimer's disease. Molecular Neurodegeneration 2011; 6:85. doi: 10.1186/1750-1326-6-85.

2. Fuentes P, Mena R. Tratamiento de la enfermedad de Alzheimer.Rol de agentes neurotróficos. Rev Chil Neuro-Psiquiat. 2009; 47 (4): 315-20.

3. Plosker G, Gauthier S. Cerebrolysin (Renacenz ${ }^{\circledR}$ ) Revisión de su uso en la demencia. Drugs Aging (español). 2009; 29(11):894-915.

4. Fernández-Verdecia C, Dáz M, Castillo-Díaz L, Álvarez-Blanco J. Neurogénesis como diana terapéutica para la enfermedad de Alzheimer. Rev Neurol. 2009; 49 (4): 193-201.

5.- Steiner E, Tata M, Frisén J. A fresh look at adult neurogenesis. Nat Med. 2019;25(4):542-3.

Rev. Med. Cine. 2020; 16(2), 135-141 Ediciones Universidad de Salamanca / @®@ J. Med. Mov., 2020; 16 (2), 135-141 